\title{
Two-dimensional electron gas at noble-metal surfaces
}

\author{
L. Bürgi ${ }^{1}$, N. Knorr ${ }^{1,2}$, H. Brune ${ }^{1}$, M.A. Schneider ${ }^{1,2}$, K. Kern ${ }^{1,2, *}$ \\ ${ }^{1}$ Institut de Physique Expérimentale, EPFL, 1015 Lausanne, Switzerland \\ ${ }^{2}$ Max-Planck-Institut für Festkörperforschung, 70569 Stuttgart, Germany
}

Received: 12 October 2001/Accepted: 23 October 2001/Published online: 3 April 2002 - @ Springer-Verlag 2002

\begin{abstract}
The electrons of the surface states on the (111) surfaces of the noble metals $\mathrm{Au}, \mathrm{Ag}$, and $\mathrm{Cu}$ form a quasitwo-dimensional (2D) free electron gas which is confined to the first few atomic layers at the crystal surface. They are scattered by the potential associated with surface defects, e.g. impurity atoms, adatoms, or step edges, leading to quantum-interference patterns in the local density of states around these defects. We have used the quantuminterference phenomena to quantitatively measure the electron phase-relaxation length and to probe long-range adsorbate interactions.
\end{abstract}

PACS: 68.37.Ef; 73.20.At; 72.10.Fk; 72.15.Lh

Because of the presence of a crystal surface, bulk forbidden electronic single-particle states may arise, leading to a band in the corresponding projected bulk band gap [1,2]. The so-called surface states are highly localized perpendicular to the surface [3]. If present, surface states may contribute a considerable fraction to the local density of states (LDOS) at and in front of the surface, and hence they can play an important role in surface physics and chemistry.

The surface states constitute a high-density low-mobility two-dimensional electron gas. Electrons in two dimensions (2D) are highly interesting from a fundamental point of view. In recent years new developments in condensed-matter physics came from two-dimensional systems, e.g. the quantum Hall effect and high-temperature superconductivity. Twodimensional systems mark the borderline between high and low dimensions, as far as localization is concerned: in one dimension, coherent back-scattering always strongly localizes the quantum states of the electrons; in three dimensions, electronic states are spatially extended (the electron "sea" of familiar metals). 2D systems are more complicated in that

*Corresponding author.

(Fax: +49-711/689-1662, E-mail: klaus.kern@fkf.mpg.de) they may show weak localization. As an illustration of the complexity of the problems in two dimensions, the nature of the zero-temperature conductance of the apparently simple system of a two-dimensional electron fluid moving in a weak random potential still poses basic challenges to our understanding [4].

There has also been a renaissance of interest in the physics of surface-state electrons, which it is argued influence a variety of physical and chemical processes at surfaces [2,5]. For example, surface states play an important role in shaping the physisorption potential, which in term determines chemical properties of surfaces, e.g. catalytic reactivity and dissociation [6-8]. Furthermore, surface states are responsible for long-range $\left(r^{-2}\right)$ substrate-mediated adsorbate interactions, which may dominate the bulk-state-mediated contribution $\left(r^{-5}\right)$ for large adsorbate-adsorbate separation [9]. Also, the contribution from surface states is relevant for the total energy balance of surface reconstructions $[2,10]$. If there is a strong interaction between electrons and phonons, the $2 \mathrm{D}$ surface state can undergo a Peierls (metal-to-insulator) transition and thereby drive a displacive surface transition [2]. As the result of such a transition a charge-density wave establishes on the surface. W (100) and Mo (100) are good examples for systems showing a surface-state charge-density wave. Occupied surface states are believed to play a role in epitaxial growth on metal surfaces, too $[8,10]$ : depopulation of the surface state is suggested to increase the diffusion barrier on terraces and to lower the activation barrier for interlayer diffusion. Since the growth mode (e.g. layer-by-layer or 3D growth) is determined by the delicate balance between intra- and interlayer diffusion, it may be tuned by specific depopulation of surface states, e.g. by adding the right surfactant. Even the equilibrium crystal shape may be influenced by surface states, through a surface-state-mediated interaction between step edges [11]. Finally, due to the fact that surface states are confined to the first few atomic layers of the crystal, they can serve as a very sensitive probe of surface magnetism, surface reconstruction, and subsurface defects [12]. It is clear from the above-mentioned examples 
that surface states may be interesting for applications, e.g. through their influence on dissociation, catalytic reactivity, or epitaxial growth.

Starting in 1991 with the work by Davis et al. [13], scanning tunneling microscopy (STM) and spectroscopy (STS) has frequently been used to investigate noble-metal surface states [14-17]. Electrons in the two-dimensional surface states are subjected to scattering at surface imperfections such as steps and point defects, leading to periodic spatial oscillations of the electronic local density of states. The standing LDOS waves are the analogue to the wellknown Friedel oscillations of the total charge density [18]. The LDOS oscillations at surfaces can be understood as interference of the electron wave traveling towards the scattering defect with the back-scattered one. The resulting quantummechanical interference patterns can be spatially resolved in scanning tunneling microscopy/spectroscopy (Fig. 1). The STM acquires a quantity roughly proportional to the surface LDOS in spectroscopic $\mathrm{d} I / \mathrm{d} V$ maps, whereas it displays the integral of the LDOS from $E_{\mathrm{F}}$ to $E_{\mathrm{F}}+e V$ in conventional topographs. Standing electron waves have been reported in both imaging modes. These STM images of the spatial distribution of the LDOS around defects enabled an unprecedented direct access to several surface electronic properties. The dispersion relation $E\left(k_{\|}\right)$of the 2D electron gas can be determined by means of STM $[16,17,19,20]$ with an accuracy comparable to state-of-the-art angle-resolved photoelectron spectroscopy studies. The advantage of STM in measuring the dispersion relation is the access to electronic states both below and above $E_{\mathrm{F}}$. Mapping of the 2D Fermi contour of surface states can be achieved through the Fourier transform of STM topographs [21] taken at very low bias voltage (inset in Fig. 1). Finally, stationary solutions of the Schrödinger equation in $2 \mathrm{D}$ were visualized for particular geometries of the scattering potential [14].

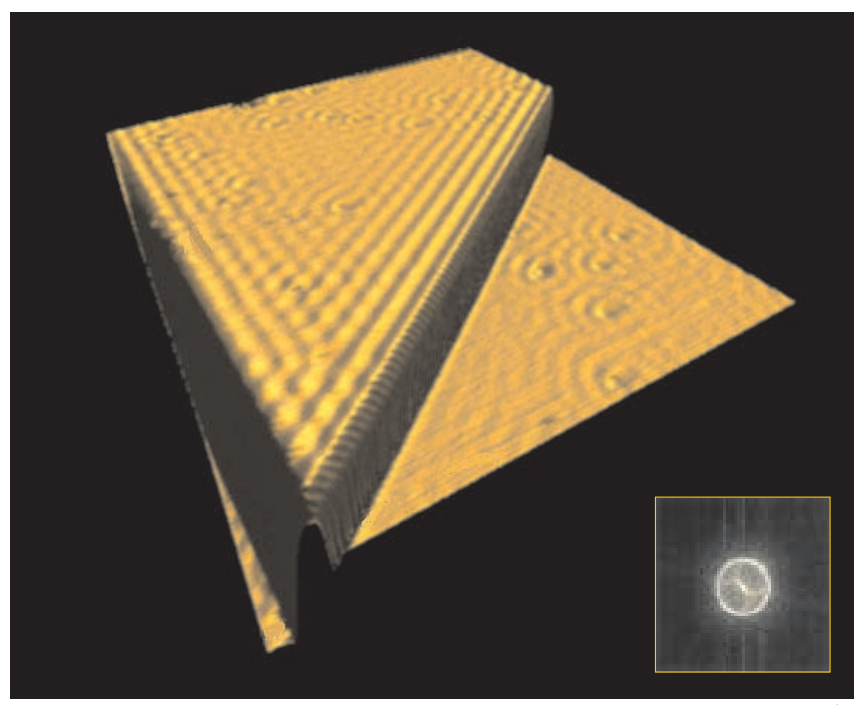

$V_{\mathrm{t}}=13 \mathrm{mV}, I_{\mathrm{t}}=0.32 \mathrm{nA}$

$1000 X 1000 \AA^{2}$

Fig. 1. Steps and defects on a $\mathrm{Cu}(111)$ surface lead to electron waves in STM constant-current images under the right conditions; image size $1000 \times 1000 \AA^{2}, V=13 \mathrm{mV}, I=0.32 \mathrm{nA}$. Analysis of these electron ripples' gives fundamental properties of the surface electrons. The inset shows a power spectrum of the Fourier transform of the STM image

\section{Surface-state band structure}

The way surface-state electrons influence STM measurements will now be discussed briefly. First of all, we recall that the density of states of the free surface-state electrons is constant above the surface-state band edge $E_{\bar{\Gamma}}$, and thus the surface density of states in the absence of surface defects such as steps and impurities is given by

$\varrho_{s}(E)=\varrho_{b}(E)+L_{0} \Theta\left(E-E_{\bar{\Gamma}}\right)$

where $\varrho_{b}(E)$ is the contribution of the bulk states, $L_{0}$ the LDOS of a free electron gas, $L_{0}=m^{*} / \pi \hbar^{2}$, and $\Theta$ a step function (only the moment states in the very center of the surface Brillouin zone are considered, where the dispersion is parabolic). From (1) one expects a step-like feature in scanning tunneling spectra. Figure 2 shows an STS spectrum taken on a clean wide $\mathrm{Ag}$ (111) terrace where interference effects due to impurity or step scattering can be neglected and thus $\varrho_{s}(E)$ is given by (1). Since for this spectrum the electric potential of the sample with respect to the tip is much smaller than the work function, the transmission factor can be assumed constant and $\mathrm{d} I / \mathrm{d} V$ is a direct measure of $\varrho_{s}(E)$ [16]. We assume a constant bulk $\left(\varrho_{\mathrm{b}}\right)$ and tip density of states $\left(\varrho_{\mathrm{t}}\right)$ in this narrow energy window. The sharp feature at $-65 \mathrm{meV}$ in Fig. 2 can thus be directly interpreted in terms of the surface-state band edge, i.e. for $E_{\bar{\Gamma}}=-65 \mathrm{meV} \mathrm{Ag} \mathrm{(111).}$ Above $-65 \mathrm{meV}, \mathrm{d} I / \mathrm{d} V$ is almost constant. This confirms the assumption of a constant $\varrho_{\mathrm{b}}$ and $\varrho_{\mathrm{t}}$. The relative contribution of the surface state to the surface density of states as measured by STS can be estimated from spectra as shown in Fig. 2, e.g. for $\operatorname{Ag}(111), L_{0} /\left(L_{0}+\varrho_{\mathrm{b}}\right) \approx 0.64$.

The contribution of surface-state electrons to the LDOS detected by the STM is about twice that of electrons from bulk states in the case of $\mathrm{Ag}$ (111). Because STM roughly measures the sample density of states at the tip apex, it is clear from Fig. 2 that the surface state contributes a considerable fraction to the LDOS in front of these noble-metal (111) surfaces. (Note that the bulk-state density decays faster into the vacuum than the surface-state density, since surfacestate electrons have a smaller average in-plane momentum.) This of course has consequences for various physical properties as described in the introduction. The feature in STS

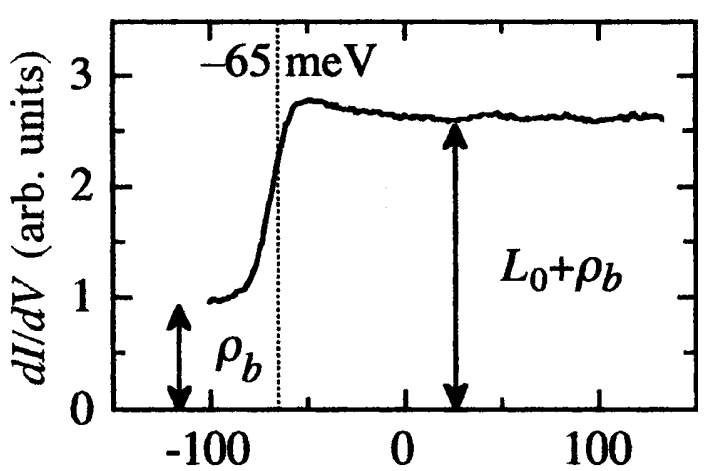

\section{$E-E_{F}(\mathrm{meV})$}

Fig. 2. Spectrum taken in the center of a clean wide $\mathrm{Ag}$ (111) terrace showing the onset of the surface state at $-65 \mathrm{meV}(T=5.9 \mathrm{~K}$, open feedback loop conditions, $\Delta V=28 \mathrm{meV}, v \approx 1 \mathrm{kHz}$, stabilizing conditions: $\left.V_{\mathrm{st}}=91 \mathrm{mV}, I=1 \mathrm{nA}\right)$ 
spectra would be the only manifestation of surface states, if the crystal surface were perfect, i.e. if there were no structural defects such as step edges and no chemical defects such as foreign surface and sub-surface atoms. But even the bestprepared surface shows residual impurities $(0.05 \%)$ and step edges separating terraces of the order of some 1000 - $\AA$ width The periodic potential experienced by the surface-state electrons can be considerably altered at and in close proximity to defects, and this naturally leads to scattering. In other terms, such impurities and step edges can act as static scatterers [22], i.e. electrons in the $2 \mathrm{D}$ surface state may be reflected elastically by such defects and thus interference effects may alter the LDOS around surface defects. The quantum-interference patterns are clearly visible in Fig. 1. The straight step edge leads to a planar wave pattern in the LDOS whereas the point defects create circular standing waves. Note the interference effects in the region between the point defect and the step edge. The oscillations in the LDOS close to defects are due to interference effects in the 2D electron gas of the surface-state electrons, analogous to Friedel oscillations in the total electron density [18]. It is clear from Fig. 1 that every static scatterer on the surface can be identified by the corresponding interference pattern. Thus STM offers the possibility to estimate the elastic mean free path of surface-state electrons, i.e. the distance an electron can travel without encountering a static scatterer, $L_{m}$, by evaluating the mean distance between such static scatterers. For our samples, with an impurity density of about $0.05 \%$, we find a global $L_{m}$ of $\sim 500 \AA$. The mobility of the surface-state electrons, defined by $\mu=e L_{m} / \hbar k_{\mathrm{F}}$, can thus be estimated to be $\mu \approx 10^{2} \mathrm{~cm}^{2} / \mathrm{Vs}$ [22]. For comparison, the mobility in a semiconductor two-dimensional electron gas can be larger than $10^{6} \mathrm{~cm}^{2} / \mathrm{Vs}$.

Since $k_{\mathrm{F}}^{3 \mathrm{D}}$ of the bulk electrons in the noble metals addressed here (about $1.2 \AA^{-1}$ ) is considerably larger than of the surface-state electrons $\left(k_{\mathrm{F}}^{2 \mathrm{D}}=0.21 \AA^{-1}, k_{\mathrm{F}}^{2 \mathrm{D}}=0.083 \AA^{-1}\right.$, and $k_{\mathrm{F}}^{2 \mathrm{D}}=0.17 \AA^{-1}$ for $\mathrm{Cu}, \mathrm{Ag}$, and $\mathrm{Au}$, respectively), and since the Thomas-Fermi screening length is inversely proportional to $k_{\mathrm{F}}$ [23], screening on these surfaces is dominated by bulk electrons. The Coulomb electric field of an electron in a surface state is thus efficiently screened by bulk electrons, and a second surface-state electron will hardly feel the presence of other surface-state electrons except through the Pauli principle. In other words, the Coulomb repulsion between electrons in the 2D gas is likely to play a minor role.

\section{Quantum coherence}

From the analysis of the quantum-interference patterns we cannot only estimate the elastic mean free path of the electrons but also determine the phase-relaxation length $L_{\phi}$, i.e. the distance a quasi-particle can propagate without losing its phase memory, which is a key quantity in solid-state physics [22]. While the mean free path is not affected by electron-electron scattering, because such processes do not lead to any loss in the net momentum, the phase-coherence length is very sensitive to phase-randomizing electronelectron collisions. The measurement of $L_{\phi}$ thus provides direct information on electron-electron interaction. In addition, quantum-mechanical interference phenomena can prevail only if $L_{\phi}$ is larger than any other relevant length scale.
Examples include Aharonov-Bohm oscillations, the quantum Hall effect, Friedel oscillations, and localization. With respect to surface physics, $L_{\phi}$ - or equivalently the lifetime of the quasi-particle $\tau_{\phi}=L_{\phi} m^{*} / \hbar^{2} k_{e V}$ - is of particular interest, since it governs the dynamics of charge transfer and electronic excitations in surface chemistry.

The approach to measure the phase-relaxation length and femtosecond lifetime of quasi-particles is based on the quantitative analysis of the amplitude decay of the quantummechanical interference patterns at step edges [24-26]. The standing waves die away within a short distance of the defect because electrons eventually scatter from one quantum state into another, destroying the pattern. So the lifetime during which an electron remains in a specific quantum state before scattering is directly reflected in the distance over which the waves persist away from a step edge.

We have measured the persistence lengths of the wave patterns as a function of temperature and bias voltage for $\mathrm{Ag}$ and $\mathrm{Cu}(111)$ by probing the thermal damping and hotelectron dynamics of these surfaces. The thermal damping of the electron standing waves is described quantitatively within a simple plane-wave model accounting for thermal broadening due to the broadening of the Fermi-Dirac distributions of sample and tip, for beating effects between electrons with different $k_{\|}$-vectors, and for inelastic collisions of the electrons, e.g. with phonons. In contrast to photoelectron spectroscopy, we measure $L_{\phi}$ close to $E_{\mathrm{F}}$ and also locally. The latter eliminates residual line widths due to surface-defect scattering embarrassing integral measurements such as photoemission. Our STM results therefore provide currently the best absolute estimates of $L_{\phi}$. Our values can be combined with photoemission results on $\mathrm{d} L_{\phi} / \mathrm{d} T$ to derive the inelastic lifetime of surface-state electrons at any $T$.

For the $\mathrm{Cu}$ and $\mathrm{Ag}(111)$ surfaces we analyzed quantitatively the spatial decay of interference patterns in the energy range $0.5-3.5 \mathrm{eV}$ above the Fermi energy and at $5.9 \mathrm{~K}$. This decay is governed by inelastic electron-electron scattering and allows for a direct determination of the corresponding lifetime of the injected quasi-particles. The determined lifetimes (1-50 fs) are shown in Fig. 3. We find a $1 /\left(E-E_{\mathrm{F}}\right)^{2}$ energy dependence of the hot-electron life-

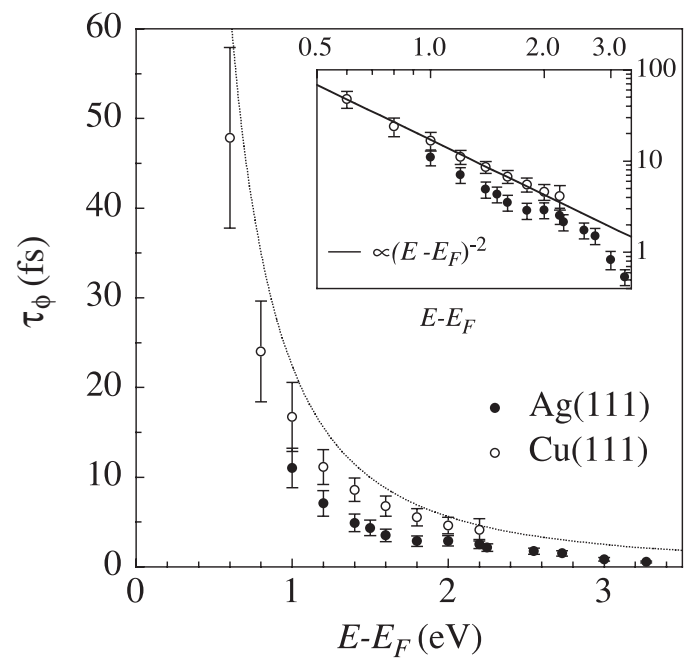

Fig. 3. Femtosecond lifetime of hot surface-state electrons on $\mathrm{Cu}$ and $\mathrm{Ag}(111)$ as a function of excess energy 
times for both $\mathrm{Ag}$ and $\mathrm{Cu}$ surface-state electrons, and our values are comparable to bulk electron lifetimes of the corresponding metals. This indicates that electronelectron interaction of hot $\mathrm{Ag}$ and $\mathrm{Cu}$ surface-state electrons with the Fermi sea is dominated by the underlying bulk electrons.

\section{Long-range adsorbate interactions}

Lateral interactions between adsorbed species have a determining influence on heterogeneous catalysis, molecular selfassembly, and thin-film epitaxy. The usually considered interactions range only a few atomic distances and have amply been studied in the past. However, for more than 20 years there has stood the theoretical prediction that there should exist adsorbate interactions of extremely long range, mediated by screening in a two-dimensional electron gas [9]. These long-range interactions were recently detected by Repp et al. [27] and by us [28] for $\mathrm{Cu}$ adatoms on the $\mathrm{Cu}$ (111) surface. We have investigated two additional adatom systems, namely $\mathrm{Co}$ on $\mathrm{Cu}(111)$ and $\mathrm{Co}$ on $\mathrm{Ag}$ (111), using low-temperature STM. Both metal substrates support a partially filled surface-state band at the Fermi energy as discussed above. The electrons in these surface states form a two-dimensional electron gas and are responsible for the interaction as predicted by theory. The interaction energy manifests itself up to $60-\AA$ distance, it decays as $1 / r^{2}$, and it oscillates with a period reflecting the surface-state band structure.

A foreign atom dissolved in a solid, or adsorbed on a surface, imposes its potential onto the host electrons which they screen by oscillations in their LDOS at the Fermi level $\left(E_{\mathrm{F}}\right)$. An example of LDOS oscillations around $\mathrm{Cu}$ atoms

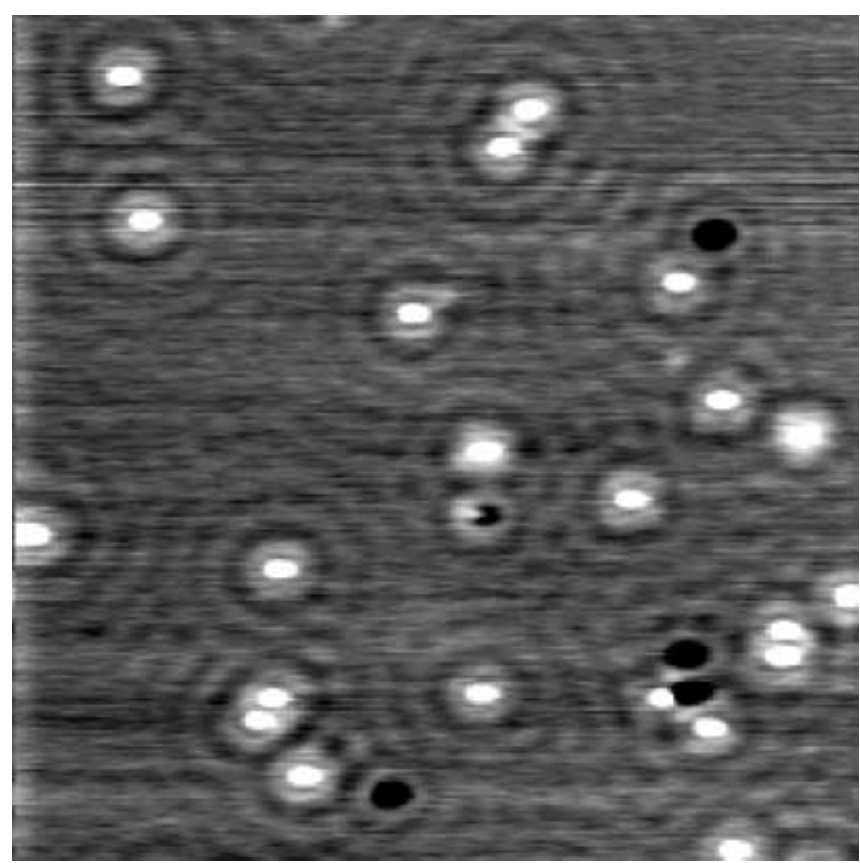

Fig. 4. A $(35 \mathrm{~nm})^{2}$ STM image of single $\mathrm{Cu}$ atoms (bright spots) on a $\mathrm{Cu}$ (111) terrace. The interference pattern in the image corresponds to the oscillations of the local density of states due to the localized scatterers $(\mathrm{Cu}$ atoms, other defects) on a $\mathrm{Cu}(111)$ substrate is given in Fig. 4. One realizes that adsorbates can interact through the fact that the adsorption energy of one adsorbate depends on the electron density, which oscillates around the other. Due to the long Fermi wavelength (e.g. $30 \AA$ for the surface state of $\mathrm{Cu}(111)$ ) these oscillatory interactions can be extremely long-ranged: Lau and Kohn predicted such oscillatory interactions to depend on distance, $r$, as $\cos \left(2 k_{\mathrm{F}} r\right) / r^{2}$ in the given experimental situation [9].

To map out the long-range interaction of the adsorbates, one has to carefully choose a temperature where diffusion is sufficiently activated so that the adatoms probe the potential in their surroundings but where they are not so fast that they are trapped by defects or steps on the surface. If these conditions are met then each recorded frame will represent an independent snapshot of the position of the adatoms. Therefore we studied the diffusion behavior of adatoms of the three mentioned systems. At low temperature, standard STM imaging (i.e. with frame rates of $1 / 100 \mathrm{~Hz}$ ) can be used to follow the diffusion of e.g. $\mathrm{Cu}$ atoms on the potential landscape of the $\mathrm{Cu}$ (111) substrate. A complete time sequence of such a diffusion study can be watched at http://www.mpistuttgart.mpg.de/KERN/Res_act/supmat_1.html, the evaluation of which results in a plot of the adatom diffusion attempt frequency against temperature, determining the diffusion barrier $E_{m}=39 \mathrm{meV}$ and the prefactor $v_{0}=10^{12} \mathrm{~Hz}$ [28].

In regions where there is a low potential, adatoms will be found more often than in regions with a high potential. Since the potential variations come from other adatoms we produced nearest-neighbor-distance histograms (Fig. 5) which show directly the oscillatory behavior of the adsorbateadsorbate interaction; there are preferred distances between adatoms and less-preferred ones. These histograms can be evaluated to directly give the interaction energy $E(r)$ as
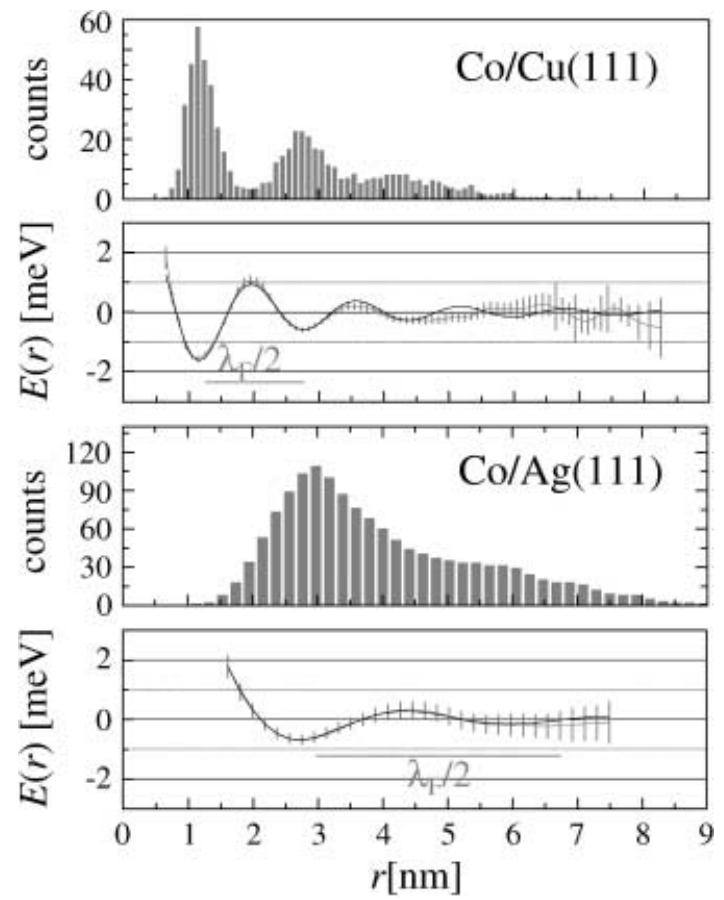

Fig. 5a,b. Nearest-neighbor-distance histograms and the resulting interaction energy as a function of distance for a $\mathrm{Co}$ on $\mathrm{Cu}(111)$ and $\mathbf{b} \mathrm{Co}$ on Ag (111) 
shown in Fig. 5. We find that $E(r)$ oscillates with a spatial dependence governed by the surface-state electrons and that its magnitude is in the $\mathrm{meV}$ range. The experimental data are in agreement with theory [29].

The electronic origin of the long-range interactions is unequivocally proven by demonstration of the scaling of the interaction length with $k_{\mathrm{F}}$. The more than two times longer wave length of the interaction on $\mathrm{Ag}$ (111) compared to $\mathrm{Cu}$ (111) is evident in Fig. 5. On the copper surface we observe a Co-Co repulsion for $r<8 \AA$ and the first minimum in interaction energy at around $12 \AA$, while at the Ag surface these values shift to $20 \AA$ and $27 \AA$, respectively. Therefore we conclude that the observed long-range interactions are indeed mediated by the nearly free 2D electron gas of the surface state.

As opposed to short-range interactions, the surface-statemediated long-range interactions are far less element-specific and therefore of general significance since they predominantly reflect the surface-state band structure. Despite the fact that the observed interaction energies are small, they are expected to influence every adsorbate/substrate system with small diffusion barriers. The substantial repulsion for dimer formation, e.g. more than $18 \mathrm{meV}$ in the case of $\mathrm{Cu} / \mathrm{Cu}(111)$ [28], can delay nucleation to much higher coverages than in classical nucleation and growth scenarios $[30,31]$. These inherent adsorbate interactions might, however, also be used in a constructive way to build selforganized artificial atomic superlattices of various species on metal substrates. These superlattices have a smaller lattice constant than any that could be prepared by other techniques and yet the atoms are far enough apart so as to not interact (i.e. bind) directly.

\section{References}

1. S.G. Davison, M. Steslicka: Basic Theory of Surface States (Oxford University Press, New York 1992)

2. E. Bertel, M. Donath: Electronic Surface and Interface States on Metallic Systems (World Scientific, Singapore 1995)

3. T.C. Hsieh, T. Miller, T.C. Chiang: Phys. Rev. Lett. 55, 2483 (1985)

4. T.M. Rice: Nature 389, 916 (1997)

5. N. Memmel, E. Bertel: Phys. Bl. 53, 323 (1997)

6. E. Bertel, P. Roos, J. Lehmann: Phys. Rev. B 52, R 14384 (1995)

7. E. Bertel: Phys. Status Solidi A 159, 235 (1997)

8. N. Memmel: Surf. Sci. Rep. 32, 91 (1998)

9. K.H. Lau, W. Kohn: Surf. Sci. 75, 69 (1978)

10. N. Memmel, E. Bertel: Phys. Rev. Lett. 75, 485 (1995)

11. N. Garcia, P. Serena: Surf. Sci. 330, L665 (1995)

12. S. Crampin: J. Phys.: Condens. Matter 6, L613 (1994)

13. L.C. Davis, M.P. Everson, R.C. Jaklevic, W. Shen: Phys. Rev. B 43, $3821(1991)$

14. M.F. Crommie, C.P. Lutz, D. Eigler: Science 262, 218 (1994)

15. P. Avouris, I.W. Lyo: Science 264, 942 (1994)

16. G. Hörmandinger: Phys. Rev. B 49, 13897 (1994)

17. J. Li, W.D. Schneider, R. Berndt: Phys. Rev. 50, 18607 (1997)

18. J. Friedel: Nuovo Cim. 7, 287 (1958)

19. Y. Hasegawa, P. Avouris: Phys. Rev. Lett. 71, 1071 (1993)

20. L. Bürgi et al.: Surf. Sci. 447, L157 (2000)

21. P.T. Sprunger et al.: Science 275, 1764 (1997)

22. S. Datta: Electronic Transport in Mesoscopic Systems (Cambridge University Press, Cambridge, UK 1995)

23. F. Stern: Phys. Rev. Lett. 18, 546 (1967)

24. O. Jeandupeux et al.: Phys. Rev. B 59, 15926 (1999)

25. L. Bürgi et al.: Phys. Rev. Lett. 82, 4516 (1999)

26. L. Bürgi et al.: J. Electron. Spectrosc. Relat. Phenom. 109, 33 (2000)

27. J. Repp et al.: Phys. Rev. Lett. 85, 2981 (2000)

28. N. Knorr, H. Brune, M. Epple, A. Hirstein, M.A. Schneider, K. Kern: Phys. Rev. B, in press (2002)

29. P. Hyldgaard, M. Persson: J. Phys.: Condens. Matter 12, L13 (2000)

30. A. Bogicevic et al.: Phys. Rev. Lett. 85, 1910 (2000)

31. K.A. Fichthorn, M. Scheffler: Phys. Rev. Lett. 84, 5371 (2000) 